\title{
Seroprevalencia de Anticuerpos para Virus Linfotrópicos Humanos (HTLV I/II) en donantes de sangre de una Clínica de Bogotá, Colombia. 1999-2004
}

\author{
Human T-lymphotropic virus (HTLV I/II) seroprevalence amongst \\ blood-donors in a hospital in Bogotá, Colombia, \\ between 1999 and 2004
}

\author{
Oscar Martínez-Nieto, Mario Isaza-Ruget, Nancy Rangel-Espinosa y \\ Olga L. Morales-Reyes
}

Clínica Colsanitas, Bogotá, D.C.Colombia. osmartin@colsanitas.com,misaza@colsanitas.com, njrangel@colsanitas.com, olmorales@colsanitas.com

Recibido 23 Junio 2006/Enviado para Modificación 11 Diciembre 2006/Aprobado 2 Mayo 2007

\section{RESUMEN}

Objetivo Determinar la prevalencia de anticuerpos para HTLV I/II, en los donantes de sangre del Banco de Sangre de la Clínica Reina Sofía, ubicada en Bogotá, Colombia.

Métodos Fueron incluidas las muestras de todos los donantes de sangre en el período comprendido entre Abril de 1999 y Agosto de 2004. A estas muestras se les realizó un ELISA para identificación de los virus HTLV I/II; a los donantes con muestras reactivas se les tomó una nueva muestra, la cual se confirmó mediante Western blot (WB). Se analizaron las encuestas de todos los donantes con pruebas reactivas para HTLV I/II en el periodo descrito y se diligenció un formulario con las variables.

Resultados La población total de donantes de sangre estudiados en los cinco años fue de 8913 donantes, 5883 (66\%) hombres y 3030 (34\%) mujeres, con un promedio de 37 años; de los cuales $26(0,3 \%)$ tuvieron ELISA doblemente reactivo para HTLV I/II y, de estos, solo seis pacientes fueron confirmados mediante WB, los cuales representan una prevalencia de $0,07 \%$

Conclusión Aunque nuestros hallazgos sugieren una baja seroprevalencia para HTLV I/II, nos permiten resaltar la importancia de implantar la prueba de detección de anticuerpos contra estos virus en todos los Bancos de Sangre del país junto con las demás pruebas que ya son indispensables según la legislación colombiana para poder suministrar sangre y hemocomponentes de calidad.

Palabras Clave: Donadores de sangre, transfusión sanguínea, bancos de sangre, Virus 1 Linfotrópico T Humano, Virus 2 Linfotrópico T Humano (fuente: DeCS, BIREME). 


\section{ABSTRACT}

Objective This study was aimed at determining anti-human T-lymphotropic virus I/II (HTLV I/II) seroprevalence amongst blood-donors at the Clínica Reina Sofía in Bogotá, Colombia, between 1999 and 2004.

Methods All people donating blood at the Clínica Reina Sofía were selected for antiHTLV I/II testing; a survey was carried out which focused on risk factors. All blood donations were screened by using enzyme immunolinked assay (ELISA); repeatedly reactive serum samples were confirmed as being HTLV I or HTLV II by using Western blot (WB).

Results 8913 blood donors, 5883 (66 \%) males and 3,030 (34\%) females having a mean age of 37 were included in the study; $26(0,3 \%)$ were repeatedly reactive in ELISA tests, $6(0,07 \%)$ of whom were confirmed by using Western blot (WB). Our findings revealed $0,07 \%$ HTLV seroprevalence amongst blood-donors, in line with other European and South-American countries.

Conclusion Although our findings suggest low HTLV I/II seroprevalence, blood-donors should be routinely screened to minimise transmission due to occult HTLV I/II infection in Colombia.

Key Words: Blood-donors, blood transfusion, blood banks, human T-lymphotropic virus 1, human T-lymphotropic virus 2 (source: $M e S H, N L M$ ).

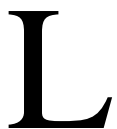

os virus linfotrópicos-T, tipo I (HTLV I) y tipo II (HTLV II) fueron los primeros retrovirus identificados en los humanos en 1980 y 1982. El virus HTLV I está involucrado como agente causal en por lo menos dos patologías, una enfermedad neurológica degenerativa, la Paraparesia Espástica Tropical /HTLV I asociada a mielopatía (PET/HAM) (1) y una neoplasia hematológica letal, la Leucemia Linfoma de células T del Adulto (LTA) (2). La infección por estos virus es endémica en el Japón, el Caribe, algunas zonas de África y Centro y Sur América (3). En poblaciones donde los virus son endémicos, la transmisión se realiza a través de relaciones sexuales, por compartir jeringas contaminadas entre drogaditos, por transfusión de componentes sanguíneos provenientes de pacientes infectados y de madre a hijo, especialmente a través de la lactancia $(4,5)$.

La transmisión de HTLV I por transfusión sanguínea o de hemocomponentes obtenidos de donantes infectados, ha sido documentada en varios estudios $(6,7)$. Por esta razón en algunos países como Estados Unidos, Francia, Holanda y Dinamarca, las autoridades sanitarias han instaurado pruebas para el tamizaje de anticuerpos contra los virus HTLV I y II aun con seroprevalencias menores a los descritas en Colombia (8-13)(Tabla 1). 
En Colombia son escasos los estudios sobre la prevalencia de esta infección y muy pocos los realizados en donantes de sangre. Según estos estudios, la seroprevalencia para HTLV I, varía dependiendo de la endemicidad de la zona, encontrando que en áreas endémicas, alcanza hasta un $3 \%$ en la población general $(14,15)$ y en áreas no endémicas la prevalencia es mucho menor $(16)$.

Tabla 1. Seroprevalencias (\%) comparativas para HTLV I y II en donantes de

\begin{tabular}{|c|c|c|c|c|c|c|c|}
\hline Colombia & Argentina & EE.UU & España & Portuqal & Francia & Italia & Grecia \\
\hline $\begin{array}{c}0,045 \dagger \\
0,45\end{array}$ & 0,05 & 0,02 & 0,004 & 0,03 & 0,003 & 0,007 & 0,02 \\
\hline Inglaterra & Suiza & Brasil & Alemania & Holanda & Dinamarca & Rumania & $\begin{array}{c}\text { Unión } \\
\text { Soviética }\end{array}$ \\
\hline 0,005 & 0,003 & $\begin{array}{c}0,04 \S \\
1,00\end{array}$ & 0,001 & 0,002 & 0,005 & 0,60 & $\begin{array}{c}0,07 \dagger \\
0,10\end{array}$ \\
\hline
\end{tabular}

A pesar del riesgo de transmisión por transfusión sanguínea, en Colombia no es obligatorio realizar tamizaje para este virus a los donantes de sangre. En nuestro país se transfunden aproximadamente 1000000 de componentes sanguíneos al año y mucho menos del $15 \%$ son tamizados para HTLV I y II (16).

De acuerdo a lo anterior, el propósito de este estudio, fue de determinar la prevalencia de anticuerpos para estos virus en donantes de sangre, en una institución localizada en una zona no endémica de Colombia y que atiende pacientes con seguridad de salud privada, durante el período comprendido entre 1999 y 2004.

\section{MÉTODOS}

Lugar: El estudio se llevó a cabo en el Banco de Sangre de la Clínica Reina Sofía, una institución de tercer nivel, ubicada en Bogotá, Colombia, área urbana considerada no endémica para esta infección. En esta institución el mayor porcentaje de pacientes atendidos corresponde a individuos con aseguramiento privado.

Diseño: Estudio de corte transversal. 
Población: Fueron incluidas las muestras de todos los donantes de sangre en el período comprendido entre Abril de 1999 y Agosto de 2004. Los candidatos a la donación de sangre se sometieron al proceso de selección de rutina a través de una encuesta y examen físico. Los elegidos para donar cumplieron con los criterios de edad (18-60 años), proceder de zonas no endémicas para la infección por HTLV I/II, gozar de buena salud y no tener factores de riesgo como infecciones, contactos sexuales de riesgo, uso de sustancias de abuso, transfusiones recientes, tatuajes, etc Los donantes con muestras inicialmente reactivas para HTLV I y II fueron llamados para tomar nueva muestra e indagar sobre factores de riesgo para dicha infección. A esta nueva muestra se le realizó nuevamente prueba de ELISA y se remitió a Estados Unidos para realizar prueba confirmatoria de Western blot (WB).

Recolección de datos: Se analizaron las encuestas de todos los donantes con pruebas reactivas para HTLV I y II en el periodo descrito y se diligenció un formulario con los datos considerados necesarios para el estudio.

Las pruebas de ELISA fueron realizadas en las instalaciones del Banco de Sangre por Bacteriólogas entrenadas y certificadas por los proveedores de los reactivos en el manejo de los equipos y montaje de las pruebas.

Se utilizó un equipo Mimiswift, totalmente automatizado y un reactivo de ELISA de Murex (ABBOTT) en el cual se utilizan microceldas cubiertas con proteínas sintéticas que representan regiones inmunodominantes de la envoltura de los virus HTLV I y II (gp 46) y una proteína recombinante transmembrana de HTLV II (gp 21) (Sensibilidad y especificidad teóricas > 99 \%). Las muestras con resultados por encima del punto de corte fueron consideradas como reactivas.

Las muestras con pruebas de ELISA doblemente reactivas se remitieron a un laboratorio de Referencia en Estados Unidos, en donde se realizó la prueba confirmatoria de WB (Cambridge Biotech Corporation, Worcester, USA). Los resultados de la pruebas de WB fueron negativos, positivos o indeterminados según los criterios establecidos por el laboratorio que desarrolla la prueba y de acuerdo con recomendaciones internacionales. $(17,18)$.

Las muestras con ELISA doblemente reactivo y con resultados de Western blot negativos o indeterminados se consideraron falsos reactivos. 


\section{RESULTADOS}

La población total de donantes de sangre estudiados durante los cinco años incluyó 8913 personas, 5883 (66 \%) hombres y 3030 (34\% mujeres), cuyas edades oscilaron entre 18 y 60 años con un promedio de 37 años.

La seroprevalencia encontrada en el período de estudio fue 0,07\%, donde se identificaron seis pacientes seropositivos para HTLV I/II, cinco hombres y una mujer, con edades entre 31 y 56 años (Tabla 2).

Tabla 2. Descripción general de la población de donantes doblemente reactivos

\begin{tabular}{|c|c|c|c|c|c|}
\hline Año & Edad & Sexo & $\begin{array}{c}\text { Absorbancia/ } \\
\text { Punto de Corte }\end{array}$ & Western Blot & Bandas Detectadas \\
\hline 1999 & SD & Masculino & 1,3 & Indeterminado & P24 \\
\hline 1999 & 29 & Masculino & 2,0 & Indeterminado & SD \\
\hline 1999 & 44 & Femenino & 2,2 & Indeterminado & SD \\
\hline 1999 & 29 & Masculino & 3,5 & Indeterminado & P28, P19 \\
\hline 1999 & 39 & Femenino & 2,2 & Negativo & Ninguna \\
\hline 1999 & 29 & Masculino & 5,0 & Indeterminado & P28, P19 \\
\hline 1999 & 39 & Femenino & 2,2 & Negativo & Ninguna \\
\hline 999 & 45 & Masculino & 1,6 & Indeterminado & P19 \\
\hline 1999 & 26 & Femenino & 2,6 & Indeterminado & P19 \\
\hline 1999 & 31 & Masculino & 2,2 & Indeterminado & P28, P19 \\
\hline 2000 & 36 & Femenino & 3,0 & Negativo & Ninguna \\
\hline 2000 & 36 & Femenino & 1,2 & Negativo & Ninguna \\
\hline 2000 & 38 & Femenino & 3,3 & Indeterminado & SD \\
\hline 2000 & 58 & Femenino & 2,1 & Negativo & Ninguna \\
\hline 2001 & 44 & Masculino & 12,3 & Positivo & $\begin{array}{l}\text { rp21ENV2+, P32, } \\
\text { P19, P24, }\end{array}$ \\
\hline 2001 & 45 & Masculino & 1,1 & Negativo & Ninguna \\
\hline 2002 & 49 & Masculino & 14,7 & Positivo & $\begin{array}{c}\text { rgp46-I, P53, gp46, P36, } \\
\text { P32, P28, P26, P24, P19, } \\
\text { gd21. }\end{array}$ \\
\hline 2002 & 20 & Femenino & 1,6 & Negativo & Ninguna \\
\hline 2002 & 56 & Masculino & 12,9 & Positivo & $\begin{array}{l}\text { rgp46-I,rgp46-II, P53, } \\
\text { gp46, P36, P32, P28, } \\
\text { P26, P24, P19, gd21. }\end{array}$ \\
\hline 2002 & 23 & Femenino & SD & Negativo & Ninguna \\
\hline 2002 & 31 & Femenino & 13,3 & Positivo & $\begin{array}{l}\text { rgp46-I, P53, gp46, P36, } \\
\text { P32,P28, P26, P24, P19, } \\
\text { gd21. }\end{array}$ \\
\hline 2003 & 35 & Masculino & 14,9 & Positivo & $\begin{array}{l}\text { rgp46-I, P53, gp46, P36, } \\
\text { P28,P26, P24, P19, gd21. }\end{array}$ \\
\hline 2003 & 40 & Masculino & 14,8 & Positivo & $\begin{array}{l}\text { rgp46-I, P53, gp46, P36, } \\
\text { P32, P28, P26, P24, P19, } \\
\text { gd21. }\end{array}$ \\
\hline 2003 & 33 & Masculino & 2,2 & Indeterminado & gd21 \\
\hline 2003 & 24 & Femenino & 2,3 & Indeterminado & gd21 \\
\hline
\end{tabular}

SD = Sin Dato; § Relación Absorbancia/Punto de Corte en Resultado de ELISA 
La determinación del tipo de HTLV según los resultados del Western blot mostró 4 casos de HTLV I, un caso de HTLV I y II y un caso en el cual la prueba de Western blot no discriminó entre I y II.

En general, los casos con resultados negativos o indeterminados por Western blot presentaron pruebas de ELISA débilmente reactivas con índices de absorbancia/punto de corte bajos (menores de 5), mientras que los casos confirmados positivos, presentaron índices de absorbancia /punto de corte altos (mayores de 12).

La banda que se observó con más frecuencia en los Western blot indeterminados fue la P19, junto con la P28 ( $\mathrm{N}=3)$ o en forma individual $(\mathrm{N}=2)$, P24 sólo se encontró en un caso. En tres de los resultados indeterminados no se tienen datos sobre las bandas.

\section{DISCUSIÓN}

Se encontró una seroprevalencia para HTLV I/II de 0,07 \% en los donantes de sangre durante los cinco años estudiados. Esta seroprevalencia es más baja que la descrita por Cortés A y cols. en el estudio de 1999 en zonas endémicas y no endémicas del país, donde identifica una prevalencia de 0,45 \% (16), Si se aplica este porcentaje de seroprevalencia general a la cifra de componentes transfundidos sin pruebas para el HTLV I/II (1 000 000), se observa que aproximadamente 3825 receptores estarán en riesgo de recibir sangre o hemocomponentes de donantes con serología positiva para estos virus cada año. En consecuencia, si tenemos en cuenta los estudios que afirman que hasta un $2 \%$ de las personas con serologías positivas pueden desarrollar alguna sintomatología relacionada ya sea con PET/HAM o LTA, teóricamente hasta 76 receptores de sangre en el país podrían estar bajo este riesgo cada año $(19,20)$.

Los resultados del presente estudio son muy similares a los obtenidos en 2005 por D'anetra y cols. en donantes de una zona no endémica; ellos identificaron en Bogotá, una seroprevalencia un poco más baja (0,045 \%) pero igualmente comparable (21).

Otro estudio de seroprevalencia en donantes de sangre por primera vez, realizado en Estados Unidos, mostró bajas prevalencias para HTLV I/II entre militares, religiosos e instituciones educativas (9,8 por 100000$)$ y mayores prevalencias entre los trabajadores de la salud (22). 
Los donantes reactivos confirmados, no fueron excluidos inicialmente en el interrogatorio que normalmente se aplica a los donantes de sangre ya que procedían del centro del país, región considerada no endémica para la infección por HTLV-I; esto minimiza el riesgo de sobrestimación de la frecuencia.

De otro lado, un encuentro importante en el estudio fue identificar que los casos positivos confirmados, tuvieron ELISA francamente reactivo con relación absorbancia/punto de corte mayor de 12, mientras que en los casos de falsos reactivos, los ELISA fueron débilmente reactivos. Este hallazgo es útil en el momento en que se suministra la consejería al donante de sangre y se toma la nueva muestra para la confirmación puesto que si se trata de una reactividad baja se puede disminuir la angustia, la ansiedad y las expectativas negativas que un resultado reactivo produce.

Debido a la diversidad de las poblaciones en las diferentes zonas geográficas, las corrientes migratorias y el desplazamiento, se considera necesario realizar más estudios en donantes de sangre en diferentes zonas del país para completar el panorama epidemiológico de esta infección en Colombia y poder estimar mejor el riesgo de transmisión de los virus HTLV I/II por transfusión sanguínea.

Los resultados de este estudio son relevantes para las personas que trabajan en los bancos de sangre y sus usuarios, puesto que reafirma que el virus no solo está presente en las zonas consideradas endémicas sino que también circula con una prevalencia importante en zonas urbanas consideradas no endémicas. Por lo tanto es necesario incluir en las encuestas a los donantes preguntas sobre riesgo específico para HTLV I/II (antecedentes o contacto con personas que presenten problemas neurológicos como parálisis, etc) así como se hace en la actualidad para HIV y hepatitis.

Los hallazgos encontrados se constituyen como un argumento a favor de implantar la prueba de detección de anticuerpos contra estos virus, para el tamizaje de donantes, en todos los Bancos de Sangre del país junto con las demás pruebas que ya son indispensables para poder colocar el sello de calidad a la sangre obtenida

Agradecimientos. Al Dr. Marco Antonio Páez por sus valiosos aportes en el desarrollo de este trabajo; al Dr. Orlando Martínez Luque por su asesoría en el diseño metodológico y muy especialmente; al grupo del Banco de Sangre de la Clínica Reina Sofía. 


\section{REFERENCIAS}

1. Gessain A, Barin F, Vernant JC, Gout O, Maurs L, Calender A, de Thé G. Antibodies to human T-lymphotropic virus type-I in patients with tropical spastic paraparesis. Lancet 1985; 2(8452): 407-410.

2. Hjelle B, Appenzeller O, Mills R, Alexander S, Torrezmartinez N, Jahnke R, Ross G. Chronic neurodegenerative disease associated with HTLV-II infection. Lancet 1992; 339: 645-646.

3. Gessain A, Mahieux R, de Thé G. Genetic variability and molecular epidemiology of human and simian T cell leukemia/lymphoma virus type I. J Acquir Immune Defic Syndr Hum. Retrovir 1996; 13 (Suppl. 1): S132-S145.

4. Schreiber GB, Murphy EL. Risk Factors for Human T-cell Lymphotropic Virus Types I and II (HTLV- I and II) in Blood Donors: The Retrovirus Epidemiology Donors Estudy. J Acquir Inmune Defic Syndr Human Retrovir 1997; 14(3): 263-271.

5. Recommendations for Counseling Persons Infected with Human T- Lymphotropic Virus, Types I y II. MMWR 1993; 42(RR-9): 1-13.

6. Murphy E, Watanabe K, Nass C, Ownby H, Williams A, Nemo G. Evidence among blood donors for a 30-year-old epidemic of human T lymphotropic virus type II infection in the United States. J Infect Dis 1999; 180:1777-1783.

7. Sullivan M, Williams A, Fang C, Notari E, Poiesz B, Ehrlich G, et al. Human Tlymphotropic virus (HTLV) types I and II infection in sexual contacts and family members of blood donors who are seropositive for HTLV type I or II. Transfusion 1993; 33: 585-590.

8. Remesar M, Mangano A, Sen L, Del Pozo A. Profile of Human T-cell lymphocytotropic virus-I/II infections in an Argentinean blood bank population. Vox Sanguinis; 2002; 83(4): 364-365.

9. Biglione M, Astarloa L, Salomón H. High Prevalence of HTLV I and HTLV II among Blood Donors in Argentina: A South American Healh Concerní. AIDS Research and Human Retroviruses 2005; 21 (1): 1-14.

10. Catalan-Soares B, Carneiro-Proietti A, Proietti F. Heterogeneus Geographic Distribution of Human T-cell Limphotropic Viruses I and II (HTLV I/II): Serological Screening Prevalence Rates in Blood Donors from Large Urban Areas in Brazil. Cad Saúde Public 2005;21(3): 926-931.

11. Taylor GP. Seroepidemiology of Human T-Cell Leukaemia/Lymphoma Viruses in Europe. J Acquir Immune Defic Syndr Hum. Retrovir 1996; 13 (1): 68-77.

12. Taylor GP. The Epidemiology of HTLV-I in Europe. Journal of Acquired Immune Deficiency Syndromes and Human Retrovirology. 1996; 13 (Suplement 1):S8S14.

13. Nübling C.M, Seifried E. Human T-cell lymphocytotropic virus prevalence in German blood donors and "at-risk" groups. Vox Sanguinis 2001; 81(3): 204-206. 
14. Zaninovic V, Moreno D, Payan C, Rodríguez A. A propósito de 5 casos de paraparesia espástica tropical en Puerto Tejada (Cauca). Colombia Médica 1997; 28:67-70.

15. Cortés A, García M. Prevalencia de marcadores para infecciones transmisibles por transfusión en donantes voluntarios. Colombia Med 1996; 27: 3-10.

16. Cortés A, Beltrán M, Gallego G Isaza L. Estudio prospectivo seroepidemiológico de infección por el Virus Linfotrópico Humano I y II (HTLV - I/II) en donantes de sangre de áreas colombianas endémicas y no endémicas. Colombia Med 1999;30: 19-25.

17. Thorstensson R, Albert J, Andersson S. Strategies for diagnosis of HTLV I and II. Transfusion 2002; 42(6): 780-791.

18. Seed Cl, Margaritis A. Improved eficiency of national HIV, HCV and HTLV antibody testing algorithsms based on sequuential screening immunoassays. Transfusion. 2003; 43(2): 226-234.

19. Murphy EL, Fridey J. HTLV - associated myelopathy in a cohort of HTLV - I and HTLV - II -infected blood donors. Neurology 1997; 48(2): 315-320.

20. Harrison LH, Vaz B, Taveira DM. Myelopathy Among Brazilians Coinfected With Human T-cell Lymphotropic Virus Type I and HIV. Neurology 1997; 48(1): 1318.

21. D’anetra V, López A, Beltrán J, Gómez ML, Rebollo S, Camacho B. Prevalencia del Virus Linfotrópico Tipo I y Tipo II en Donantes de Sangre en Zona no Endémica. Colombia. Revista Argentina de Transfusión 2005;31(3):95.

22. Wang B, Schreiber GB. Prevalence of transfusion-transmissible viral infections in first-time US blood donors by donation site. Transfusion; 43(6): 705-712. 